Original article

\title{
Long-term effects of folic acid and vitamin-B12 supplementation on fracture risk and cardiovascular disease: Extended follow-up of the B- PROOF trial
}

\author{
Sadaf Oliai Araghi a, b, *, Jessica C. Kiefte-de Jong b, c, Suzanne C. van Dijk ${ }^{\text {a, d }}$, \\ Karin M.A. Swart ${ }^{\mathrm{e}}$, Kim J. Ploegmakers ${ }^{\mathrm{f}}$, M. Carola Zillikens ${ }^{\mathrm{a}}$, Natasja M. van Schoor ${ }^{\mathrm{g}}$, \\ Lisette C.P.G.M. de Groot ${ }^{\text {h }}$, Paul Lips ${ }^{\mathrm{i}}$, Bruno H. Stricker ${ }^{\mathrm{b}}$, André G. Uitterlinden ${ }^{\mathrm{a}}{ }^{\mathrm{b}}$, \\ Nathalie van der Velde ${ }^{\mathrm{a}, \mathrm{f}}$ \\ a Department of Internal Medicine, Erasmus University Medical Center, Rotterdam, the Netherlands \\ ${ }^{\mathrm{b}}$ Department of Epidemiology, Erasmus University Medical Center, Rotterdam, the Netherlands \\ ${ }^{c}$ Department of Public Health and Primary Care, Leiden University Medical Center/LUMC Campus, The Hague, the Netherlands \\ ${ }^{\mathrm{d}}$ Department of Geriatric Medicine, Franciscus Gasthuis \& Vlietland, Schiedam, the Netherlands \\ e Amsterdam UMC, Vrije Universiteit Amsterdam, Department of General Practice and Elderly Care Medicine, Amsterdam Public Health Research Institute, \\ Amsterdam, the Netherlands \\ ${ }_{\mathrm{f}}^{\mathrm{f}}$ Amsterdam UMC, (University) of Amsterdam, Section of Geriatric Medicine, Department of Internal Medicine, Amsterdam Public Health Research Institute, \\ Amsterdam, the Netherlands \\ ${ }^{\mathrm{g}}$ Amsterdam UMC, Vrije Universiteit Amsterdam, Department of Epidemiology and Biostatistics, Amsterdam Public Health Research Institute, Amsterdam, \\ the Netherlands \\ ${ }^{\mathrm{h}}$ Division of Human Nutrition and Health, Wageningen University, Wageningen, the Netherlands \\ ${ }^{\mathrm{i}}$ Amsterdam UMC, Vrije Universiteit Amsterdam, Department of Internal Medicine, Endocrine Section, Amsterdam Public Health Research Institute, \\ Amsterdam, the Netherlands
}

\section{A R T I C L E I N F O}

\section{Article history:}

Received 8 January 2020

Accepted 29 July 2020

\section{Keywords:}

B-vitamins

Fracture

Cardiovascular disease

Long-term follow-up

\section{S U M M A R Y}

Background \& aims: In the initial B-proof, we found inconsistent results of B vitamin supplementation. However, the debate regarding the effects of B vitamins on age-related diseases continues. Therefore, our aim was to investigate the long-term effects (5-7 years follow-up) of an intervention with folic acid and vitamin-B12 supplementation on fracture and cardiovascular disease risk.

Methods: Extended follow-up of the B-PROOF trial, a multi-center, double-blind randomized placebocontrolled trial designed to assess the effect of 2-3 years daily supplementation with folic acid $(400 \mu \mathrm{g})$ and vitamin-B12 $(500 \mu \mathrm{g})$ versus placebo $(n=2,919)$. Primary outcome was verified selfreported fracture incidence and secondary outcomes were self-reported cardiovascular endpoints, which were collected through a follow-up questionnaires Proportional hazard analyses was used for the effect of the intervention on risk of fracture(s) and logistic regression for the effect of the intervention on risk of cardiovascular disease.

Results: A total of 1,298 individuals (44.5\%) participated in the second follow-up round with median of 54 months [51-58], ( $n=662$ and $n=636$, treatment versus placebo group). Median age at baseline was 71.0 years [68.0-76.0] for both groups. No effect was observed of the intervention on osteoporotic fracture or any fracture risk after a follow-up (HR: 0.99, 95\% CI: $0.62-1.59$ and HR: $0.77 ; 95 \%$ CI: 0.50 -1.19 , respectively), nor on cardiovascular or cerebrovascular disease risk (OR: $1.05 ; 95 \% \mathrm{CI}$ : $0.80-1.44$ and OR: 0.85 ; $95 \% \mathrm{CI}$ : $0.50-1.45$, respectively). Potential interaction by baseline homocysteine concentration was observed for osteoporotic- and any fracture ( $p=0.10$ and 0.06 respectively), which indicated a significantly lower risk of any fracture in the treatment group with higher total homocysteine concentrations $(>15.1 \mu \mathrm{mol} / \mathrm{l})$. No age-dependent effects were present.

Conclusions: This study supports and extends previous null-findings of the B-PROOF trial and shows that supplementation of folic acid and vitamin-B12 has no effect on fracture risk, nor on cardiovascular disease in older individuals over a longer follow-up period. However, B-vitamin supplementation may be

Abbreviations: B-PROOF, B-vitamins for the prevention of osteoporotic fractures; GPs, general practitioners; BMD, bone mineral density; CVD, cardiovascular disease; Hcy, homocysteine; HoloTC, holotranscobalamin; MI, myocardial infarction; AP, angina pectoris; CVA, cerebrovascular accident; TIA, transient ischemic attack.

* Corresponding author. Department of Internal Medicine and Epidemiology, Erasmus University Medical Center, Rotterdam, the Netherlands.

E-mail address: s.oliaiaraghi@erasmusmc.nl (S. Oliai Araghi). 
beneficial in reducing fractures in individuals with high total homocysteine concentrations, a finding which needs to be replicated.

(C) 2020 The Author(s). Published by Elsevier Ltd. This is an open access article under the CC BY license (http://creativecommons.org/licenses/by/4.0/).

\section{Introduction}

Previously, homocysteine-lowering therapy has been suggested as a potential treatment option for common diseases such as osteoporosis and cardiovascular disease [1]. An effective method of normalizing homocysteine concentration is treatment with B-vitamins, which play a central role in homocysteine metabolism [2]. Over the years, several intervention trials have been performed, but conflicting effects of treatment with B-vitamins and homocysteine were observed for both fracture and cardiovascular outcomes $[3,4]$.

An association between increased homocysteine concentration and risk of cardiovascular disease and fracture has been observed in particular in older individuals [5]. Recently within the older BPROOF population, we observed no effect of B-vitamin intervention on the overall incidence of coronary heart disease, but a significantly but slightly lower risk of cerebrovascular events was observed among females. This was further confirmed by a recent meta-analysis that also showed a reduced risk of stroke with folic acid alone and B-complex supplementation [5]. Yet, this metaanalysis included, beside our initial B-PROOF study, studies with mostly younger individuals which makes it difficult to extrapolate these findings to older populations. With regard to fractures, the BPROOF trial did show lower osteoporotic fracture incidence but only in a subgroup of compliant persons aged 80 years and over [6]. The mechanism of B-vitamins in bone health is not yet completely understood, but B-vitamins appear to influence the development of collagen and alter the metabolism of osteoblasts in a dosedependent manner [7,8]. Moreover, low levels of B-vitamins have been associated with low bone mineral density (BMD) and increased fracture risk [9]. The recent meta-analysis by Gracia Lopez et al., however, did not show a significant reduction of fractures after B-vitamin supplementation [10]. From all included RCT's in this meta-analysis with different dosages of B-vitamins, only our initial B-PROOF trial had fractures as main outcome and included older participants [6]. Other trials which included selected patients with cardiovascular disease or colorectal adenomas, found no significant differences in fracture risk (as secondary outcome) between the groups [4,11-13].

Another potential explanation for previous inconsistent and null-findings may be the relatively short follow-up time of the trials. Besides increasing power, prolonged follow-up could account for the potential latency period between exposure (B-vitamins) till event. As known, the latency period of coronary heart disease (from exposure to mortality) may be 10 years or more [14]. Thus, additional post-trial follow-up on risk of fractures and cardiovascular diseases could provide valuable scientific information on potential long-term effects of supplementation [15]. For that reason, this study aimed to examine the primary and secondary endpoints of the B-PROOF study (fracture and cardiovascular disease) with a longer follow-up time to validate our previous findings [16].

\section{Materials and methods}

The initial B-vitamins for the Prevention Of Osteoporotic Fractures (B-PROOF) trial was a multi-center (Erasmus MC Rotterdam, VU University Medical Center Amsterdam (VUmc) and Wageningen University (WUR), the Netherlands), double blinded, randomized placebo-controlled study. This trial was designed to investigate the effect of daily oral folic acid (400 $\mu \mathrm{g})$ and vitaminB12 $(500 \mu \mathrm{g})$ supplementation (treatment group) on fracture incidence as a primary outcome. Secondary outcomes included amongst others cardiovascular events. Placebo- and treatment group received daily $15 \mu \mathrm{g}$ (600 IU) vitamin D3 to ensure a normal vitamin D status. The duration of the intervention was 2 years, and this was extended in a subgroup for 1 more year with the aim to increase power ( $n=339$ out of 2,919 participants had 3 years intervention) [17].

The recruitment period was between September 2008 and March 2011. A total of 2,919 participants aged 65 years and over with an elevated homocysteine concentration (12-50 $\mu \mathrm{mol} / \mathrm{l})$ were included in the study. Exclusion criteria were renal insufficiency (creatinine level > $150 \mu \mathrm{mol} / \mathrm{l}$ ), history of malignancy (except nonmelanoma skin cancer) in the past 5 years before the recruitment and use of high dosage of B-vitamins (folic acid intake $>300 \mu \mathrm{g} /$ day and/or intramuscular injections of vitamin-B12 at recruitment and baseline). The study protocol of the trial and a detailed description of the study have been reported elsewhere [17]. The B-PROOF study was registered in the Netherlands Trial Register (NTRNTR1333) and ClinicalTrials.gov (NCT00696514). The study protocol was approved by The Medical Ethics committees of Erasmus MC, VUmc and WU universities [17]. In 2015, we extended the follow-up of the original B-PROOF study by sending the participants who gave permission to contact them additional questionnaires to investigate the longterm effect of the intervention on risk of cancer, fracture and cardiovascular diseases $(n=1,298)$. The long-term effect on cancer has been described in a separate paper [18]. The current paper describes the outcomes of the extended follow-up on fracture and cardiovascular diseases. End of follow-up for this study was December of 2017.

\subsection{Covariates and outcomes}

A wide set of measurements (e.g. BMI, medical history, plasma homocysteine (Hcy), serum folate, vitamin-B12, holotranscobalamin (HoloTC), methylmalonacid (MMA) and 25(OH) D) was performed at baseline and at 2-y follow-up [17]. The extended (5-7y) follow-up structured questionnaire was used to assess self-reported medical history (fractures and cardiovascular disease (CVD)), alcohol intake and smoking habits. All reported fractures were verified with the general practitioner of the participants and were categorized as osteoporotic (all fractures excluding head, hand, finger, foot or toe fractures, fractures caused by traffic accidents or by cancer) or any fracture. CVD was assessed, in concordance with the original B-PROOF trial outcomes, as self-reported and without date of event. Self-reported CVD events were also verified by the GP in order to obtain information on the validity of these events. Cohen's kappa coefficient was calculated for the agreement between the self-reported and verified CVD events. For CVD the coefficient was 0.89 (excellent agreement between self-reported and verified events) and for Cerebrovascular accident (CVA) 0.72 (fair to good agreement between self-reported and verified events). CVD was classified as any type of CVD, and subgroups of myocardial infarction (MI), angina pectoris (AP), hearth failure and cardiac valve disease were assessed. CVA and Transient Ischemic Attack (TIA) were included as cerebrovascular disease. 


\subsection{Statistical analysis}

Mean with standard deviations (SD), or median with interquartile range (IQR) or percentages were reported. Differences between groups at baseline were tested with t-test for continuous variables and Mann-Whitney $U$ for not normally distributed data. Chi-squared tests were used for categorical variables. Kaplan-Meier event curve was used for the cumulative event-free survival on the basis of fracture incidence. The follow-up time was calculated as the number of months from the baseline measurement until the first diagnosis of incident fracture, death (derived from the national institute 'Centrum voor familiegeschiedenis', CBG), loss-to-follow-up, or end of the study period, whichever occurred first for the participants with complete follow-up. The incidence rate ratio was calculated on the incidence-rate of fracture for both treatment groups, which is defined as the number of events divided by the total sum of the follow-up in each group (cases/persons years). Participants were analysed based on the initial treatment allocation (intention to treat principle (ITT)). Unadjusted Cox proportional hazard analyses were conducted with treatment (treatment vs. placebo group) as the independent variable and the fracture diagnosis as the dependent outcome variable. Subsequently, per protocol (PP) analyses were performed that included data only from participants who were compliant during the intervention ( $>80 \%$ of pills consumed) [17]. Then, cluster proportional hazard model was used to analyse the effect of the intervention on the multiple osteoporotic fracture risk [19]. Multiple osteoporotic fracture was defined as total osteoporotic fractures during the intervention in the total population.

For the analyses with cardiovascular and cerebrovascular disease as outcome, binary logistic regression analysis was used for the population with complete follow-up, due to the absence of a precise time of onset, a Cox regression analysis was not possible. Selfreported events were used as the dependent factor and the treatment group as the independent factor. Multivariable Cox proportional hazard regression analyses and binary logistic regression analysis were adjusted for serum HoloTC since this variable differed significantly between the treatment and the placebo group regardless of randomization. All other potential confounders were equally distributed between both groups. Furthermore, as in the original BPROOF trial, multivariable analyses were performed to test the interaction between sex, age (continuous) study center, homocysteine concentration, MTHFR polymorphism, vitamin-B12 and folate level and baseline CVD. Appropriate subgroup analyses were performed when $p$ for interaction was $<0.10$.

$p$-Values $<0.05$ were considered to be statistically significant (except the interaction analysis). Analyses were performed using IBM SPSS 24, the library survival R statistical packages [20].

\section{Results}

The baseline characteristics of the total population (initial BPROOF trial with 2-3 years follow-up, $n=2,919)$ and population with ( $n=1,298)$ and without $5-7$ years follow-up $(n=1,621)$ are shown in Table 1 . The median or mean values for all variables were similar for treatment $(n=1,485)$ and placebo $(n=1,461)$ group at baseline, except for serum HoloTC levels, with higher levels in the treatment group compared to placebo group (65.0 [48.0-86.0] vs. $63.0[45.0-84])$. A total of 1,298 participants $(n=662$ in the treatment group and $n=636$ in the placebo group) sent the second follow-up questionnaire back, with the median age at baseline of 71.0 years [68.0-76.0] for both groups. The median follow-up time was 54 months [IQR 51-58]. There were some differences in the baseline characteristics of the participants who did not return the second questionnaire compared to the participants with 5-7 y follow-up. For example, the non-responders were older, with a higher percentage of women with median age at baseline of 75.0 years [70.0-80.0] for the treatment group $(n=799)$ and 74.0 years [70.0-80.0] for the placebo group $(n=822)$ (Table 1$)$. Furthermore, the baseline characteristic of the participants with 5-7 y follow-up was different for vitamin B12 level between placebo and the treatment group ( $15.7 \%$ vs. $15.0 \%$, respectively).

\subsection{Fracture incidence}

Table 2 shows the incidence of osteoporotic fractures, any fractures, and the effect of the intervention on these outcomes. The incidence of osteoporotic fractures was not different between the treatment and placebo group for participants with complete followup ( $n=1,298,35$ vs. 35). However, the incidence of any fractures tended to be lower in the treatment group compared to the placebo group ( 37 vs. $47, p=0.19$ ). The results of ITT analyses of the effect of folic acid and vitamin B-12 on verified first osteoporotic fractures in participants with complete follow-up showed no effect of treatment of folic acid and vitamin-B12 on first osteoporotic fracture and any fracture risk after a follow-up of 5-7 years in the adjusted model (HR: 0.99, 95\% CI: 0.62; 1.59 and HR: 0.77, 95\% CI: 0.50; 1.19 respectively, Table 2 and Fig. 2). PP analyses showed also no effect of the treatment of folic acid and vitamin-B12 on both osteoporotic fractures and any fractures in the final model (HR: 1.09, 95\% CI: 0.66-1.79 and HR: 0.81, 95\% CI: 0.52-1.28 respectively, Table 2).

Total fractures and first osteoporotic fractures from baseline to the end of follow-up periods for the total population (participants with only FU1) and participants with complete follow-up (participants with FU1 and FU2), are shown in Table S1. Table S3 shows the number of osteoporotic fractures in the treatment and placebo group at FU1 and FU2 in detail. In Table 2, the effect of the intervention on the multiple osteoporotic fracture risk is shown. In the total population, the number of osteoporotic fractures was lower in the treatment group than in the placebo group ( $81 \mathrm{vs.} 86$ ). The intervention had no effect on multiple osteoporotic fracture incidence in the total population ( $\mathrm{HR}=0.93 ; 95 \% \mathrm{CI}$ : 0.65-1.33, Table 2$)$.

In the interaction analysis, we found only a significant interaction term for homocysteine concentration for the effect of the intervention on first verified osteoporotic and any fracture ( $p=0.10$ and $p=0.06$ respectively). After stratification for homocysteine in tertiles, the risk of osteoporotic fracture did not significantly differ between the groups. However, we found a lower risk of any fractures for those with homocysteine concentration above $15.1 \mathrm{mmol} / \mathrm{l}$ for the treatment group compared to the placebo group (HR: 0.42; $95 \%$ CI: $0.19-0.92$, Fig. 1). Baseline characteristics of population with homocysteine concentration $<$ and $\geq 15.1 \mathrm{mmol} / \mathrm{l}$ are shown in Table S2. Besides, we found differences in baseline characteristics between placebo and intervention group within each category by homocysteine level. For the participants with homocysteine concentration $<15.1 \mathrm{mmol} / \mathrm{l}$ (lower two tertiles), weight $(p=0.04$ ), vitamin B12 level $(p=0.01)$, smoking $(p=0.03)$ and kidney problems $(p=0.04)$ were different between placebo and intervention group. For the participants with homocysteine $\geq 15.1$ (higher tertile), there were no differences between placebo and intervention group in baseline characteristics.

Median homocysteine concentration for the participants with the extended follow-up changed more after the intervention in the treatment group in the highest tertile compared to the first two tertiles $(-3.1$ for the first tertile and $-4.1 \mathrm{mmol} / \mathrm{l}$ for the second tertile, -6.0 for the last tertile, data not shown).

The interaction analysis of the effect of the intervention on multiple fracture incidence showed no significant interaction terms for age, gender, study center and total homocysteine and MTHFR (data not shown). 
Table 1

Baseline characteristics of total study population $(n=2,919)$ and the participants with $(n=1,298)$ and without $(1,621)$ follow-up.

\begin{tabular}{|c|c|c|c|c|c|c|}
\hline & \multicolumn{2}{|c|}{$\begin{array}{l}\text { Populatio } \\
\text { n with } 2-3 Y \text { FU }(n=2,919)\end{array}$} & \multicolumn{2}{|c|}{$\begin{array}{l}\text { Population with } \\
5-7 Y \text { FU }(n=1,298)\end{array}$} & \multicolumn{2}{|l|}{$\begin{array}{l}\text { Population } \\
\text { without 5-7Y FU } \\
(n=1,621) \\
\end{array}$} \\
\hline & $\begin{array}{l}\text { Placebo } \\
(n=1,458)\end{array}$ & $\begin{array}{l}\text { Treatment } \\
\text { group }(n=1,461)\end{array}$ & $\begin{array}{l}\text { Placebo } \\
(n=636)\end{array}$ & $\begin{array}{l}\text { Treatment } \\
\text { group }(n=662)\end{array}$ & $\begin{array}{l}\text { Placebo } \\
(n=822)\end{array}$ & $\begin{array}{l}\text { Treatment } \\
\text { group }(n=799)\end{array}$ \\
\hline Age (years) ${ }^{b}$ & $73.0[69.0 ; 78.0]$ & $73.0[69.0 ; 78.0]$ & $71.0[68.0-76.0]$ & $71.0[68.0-76.0]$ & $75.0[70.0-80.0]$ & $74.0[70.0-80.0]$ \\
\hline Sex (\%women) & 49.7 & 50.4 & 43.9 & 46.1 & 54.1 & 53.9 \\
\hline \multicolumn{7}{|l|}{ Study center (\%) } \\
\hline WU & 29.6 & 29.2 & 23.7 & 20.7 & 34.1 & 36.2 \\
\hline VUmc & 26.8 & 26.4 & 23.4 & 24.3 & 29.4 & 28.2 \\
\hline EMC & 43.6 & 44.4 & 52.8 & 55.0 & 36.5 & 35.7 \\
\hline \multicolumn{7}{|l|}{ Education years (\%) } \\
\hline Low & 53.6 & 52.4 & 47.8 & 48.6 & 57.9 & 55.6 \\
\hline Intermediate & 21.1 & 21.1 & 22.5 & 21.2 & 20.0 & 21.0 \\
\hline High & 25.4 & 26.5 & 29.7 & 30.2 & 22.1 & 23.4 \\
\hline Height $(\mathrm{cm})^{\mathrm{a}}$ & $169.2(9.3)$ & $169.4(9.4)$ & $170.6(9.1)$ & $170.6(9.0)$ & $168.1(9.3)$ & $168.3(9.6)$ \\
\hline Weight $(\mathrm{kg})^{\mathrm{a}}$ & $77.8(13.3)$ & $77.9(13.3)$ & $78.7(12.0)$ & $77.0(13.6)$ & $77.2(14.2)$ & $77.0(13.6)$ \\
\hline $\operatorname{BMI}\left(\mathrm{kg} / \mathrm{m}^{2}\right)^{\mathrm{a}}$ & $27.2(4.0)$ & $27.1(4.0)$ & $27.0(3.5)$ & $27.1(3.8)$ & $27.3(4.3)$ & $27.2(4.1)$ \\
\hline \multicolumn{7}{|l|}{ Alcohol consumption (\%) } \\
\hline Light & 66.8 & 68.0 & 74.1 & 66.4 & 69.0 & 70.2 \\
\hline Moderate & 29.0 & 28.5 & 22.9 & 28.9 & 26.9 & 26.4 \\
\hline Excessive & 4.2 & 3.5 & 2.9 & 4.7 & 4.2 & 3.4 \\
\hline \multicolumn{7}{|l|}{ Smoking status (\%) } \\
\hline Current (cigarette) & 9.7 & 9.5 & 8.0 & 9.2 & 11.1 & 9.8 \\
\hline Homocysteine $(\mathrm{mmol} / \mathrm{l})^{\mathrm{b}}$ & $14.4[13.0-16.7]$ & $14.3[13.0-16.5]$ & $14.1[12.9-16.0]$ & $14.0[12.8-15.9]$ & $14.8[13.2-17.2]$ & $14.6[13.2-17.2]$ \\
\hline HoloTC $(\mathrm{pmpl} / \mathrm{l})^{\mathrm{b}}$ & $63.0[45.0-84.0]$ & $65.0[48.0-86.0]$ & $65.0[46.5-87.0]$ & $66.0[51.0-87.5]$ & $60.0[44.0-82.25]$ & $62.0[45.0-84.75]$ \\
\hline $25(\mathrm{OH}) \mathrm{D}(\mathrm{nmol} / \mathrm{l})^{\mathrm{a}}$ & $55.8(23.9)$ & $55.5(25.8)$ & $57.5(23.9)$ & $55.9(24.9)$ & $54.5(23.9)$ & $55.2(26.5)$ \\
\hline Vitamin-B12 (pmol/l) ${ }^{\mathrm{a}}$ & $282.3(114.0)$ & $288.6(117.9)$ & $287.8(119.9)$ & $283.9(102.3)$ & $278.1(109.0)$ & $292.4(129.2)$ \\
\hline $\operatorname{MMA}(\operatorname{mcgmol} / 1)^{\mathrm{a}}$ & $0.3(0.4)$ & $0.3(0.2)$ & $0.3(0.2)$ & $0.3(0.2)$ & $0.3(0.5)$ & $0.3(0.2)$ \\
\hline Folate $(\mathrm{nmol} / \mathrm{l})^{\mathrm{a}}$ & $20.7(8.7)$ & $21.4(13.9)$ & $20.9(8.1)$ & $21.4(10.0)$ & $20.5(9.1)$ & $21.4(16.5)$ \\
\hline Folic acid supplement use (\% yes) & 14.8 & 14.0 & 14.7 & 13.9 & 14.9 & 14.1 \\
\hline Vitamin-B12 supplement use (\% yes) & 15.2 & 16.4 & 15.7 & 15.0 & 15.6 & 15.5 \\
\hline Medication use (\% yes) & 84.3 & 83.9 & 81.3 & 82.0 & 86.6 & 85.4 \\
\hline Falls frequency (12 months before baseline) & 32.5 & 32.6 & 30.1 & 31.2 & 34.3 & 33.6 \\
\hline History of fracture (\% yes) & 42.9 & 41.3 & 40.3 & 60.3 & 45.0 & 57.3 \\
\hline Cardiovascular diseases (\%) & 19.1 & 18.6 & 21.5 & 21.0 & 17.3 & 16.6 \\
\hline
\end{tabular}

FU = Follow-up, MMA = methylmalonacid.

${ }^{\text {a }}$ Mean (SD).

b Median (IQR)

Table 2

Effect of folic acid and vitamin-B12 intervention on verified first osteoporotic fracture in participants with complete follow-up ( $n=1298$ ).

\begin{tabular}{|c|c|c|c|c|c|c|}
\hline \multirow[t]{2}{*}{ Outcome } & \multicolumn{2}{|c|}{ Placebo group } & \multicolumn{2}{|c|}{ Treatment group } & \multicolumn{2}{|l|}{$5-7$ years $\mathrm{FU}$} \\
\hline & $n$ & Cases/100 PY & $n$ & Cases/100 PY & $\mathrm{HR}[95 \% \mathrm{CI}]^{\mathrm{a}}$ & $\mathrm{HR}[95 \% \mathrm{CI}]^{\mathrm{b}}$ \\
\hline \multicolumn{7}{|c|}{ ITT analysis ( $n=1298: 636$ in placebo and 662 in control group $)^{c}$} \\
\hline Osteoporotic fractures & 35 & 1.3 & 35 & 1.2 & $0.96[0.60 ; 1.53]$ & $0.99[0.62 ; 1.59]$ \\
\hline Any fractures & 47 & 1.7 & 37 & 1.3 & $0.75[0.49 ; 1.16]$ & $0.77[0.50 ; 1.19]$ \\
\hline \multicolumn{7}{|c|}{ PP analysis ( $n=12451325$ in placebo and 1336 in control group $)^{c}$} \\
\hline Osteoporotic fractures & 30 & 1.1 & 33 & 1.2 & $1.04[0.64 ; 1.71]$ & $1.09[0.66 ; 1.79]$ \\
\hline Any fractures & 42 & 1.6 & 35 & 1.2 & $0.79[0.51 ; 1.24]$ & $0.81[0.52 ; 1.28]$ \\
\hline \multicolumn{7}{|c|}{ Multiple fracture incidence analysis ( $n=29191458$ in placebo and 1461 in control group) ${ }^{\mathrm{d}}$} \\
\hline Osteoporotic fractures & 86 & NA & 81 & NA & $0.92[0.65 ; 1.31]$ & $0.93[0.65 ; 1.33]$ \\
\hline
\end{tabular}

${ }^{*} p<0.05$. HR = Hazard Ratio; ITT = Intention To Treat; PP = Per Protocol; PY = person years, FU = Follow-up.

a Unadjusted model.

b Adjusted model for HoloTC (significant difference between treatment and placebo group).

c Values were derived from Cox proportional hazards, ITT, and PP analyses.

d Values were derived from Cluster proportional hazards.

\subsection{Cardiovascular disease and cerebrovascular disease}

The incidence of any type CVD, MI, AP, heart failure and/or cardiac valve disease during the follow-up period for the participants with complete follow-up was higher in the treatment group compared to the placebo group (130 vs. 120,20 vs. 18,43 vs. 42 and 41 vs. 35 respectively), except for cerebrovascular disease (27 vs. 30). However, the differences were not statistically significant (logistic regression analysis, Table 3 ). Sex and study center were significant modifiers in the intervention effect on any type of CVD ( $p$-for interaction $=0.03$ and 0.09 , respectively). In addition, sex had a potential interaction with the intervention effect on heart failure/cardiac valve disease ( $p$-for interaction $=0.10$ ). After stratification for sex, the intervention showed a higher risk of any type of CVD, heart failure and/or cardiac valve disease for women compared to men, but these differences were non-significant (for women: $\mathrm{OR}=1.53 ; 95 \% \mathrm{CI}$ : $0.99-2.35$ for any type of CVD and OR $=1.52 ; 95 \% \mathrm{CI}: 0.78-2.97$ for heart failure and/or cardiac valve disease, for men: $\mathrm{OR}=0.81$; 95\% CI: $0.56-1.17$ for any type of CVD and $\mathrm{OR}=0.71 ; 95 \% \mathrm{CI}$ : 


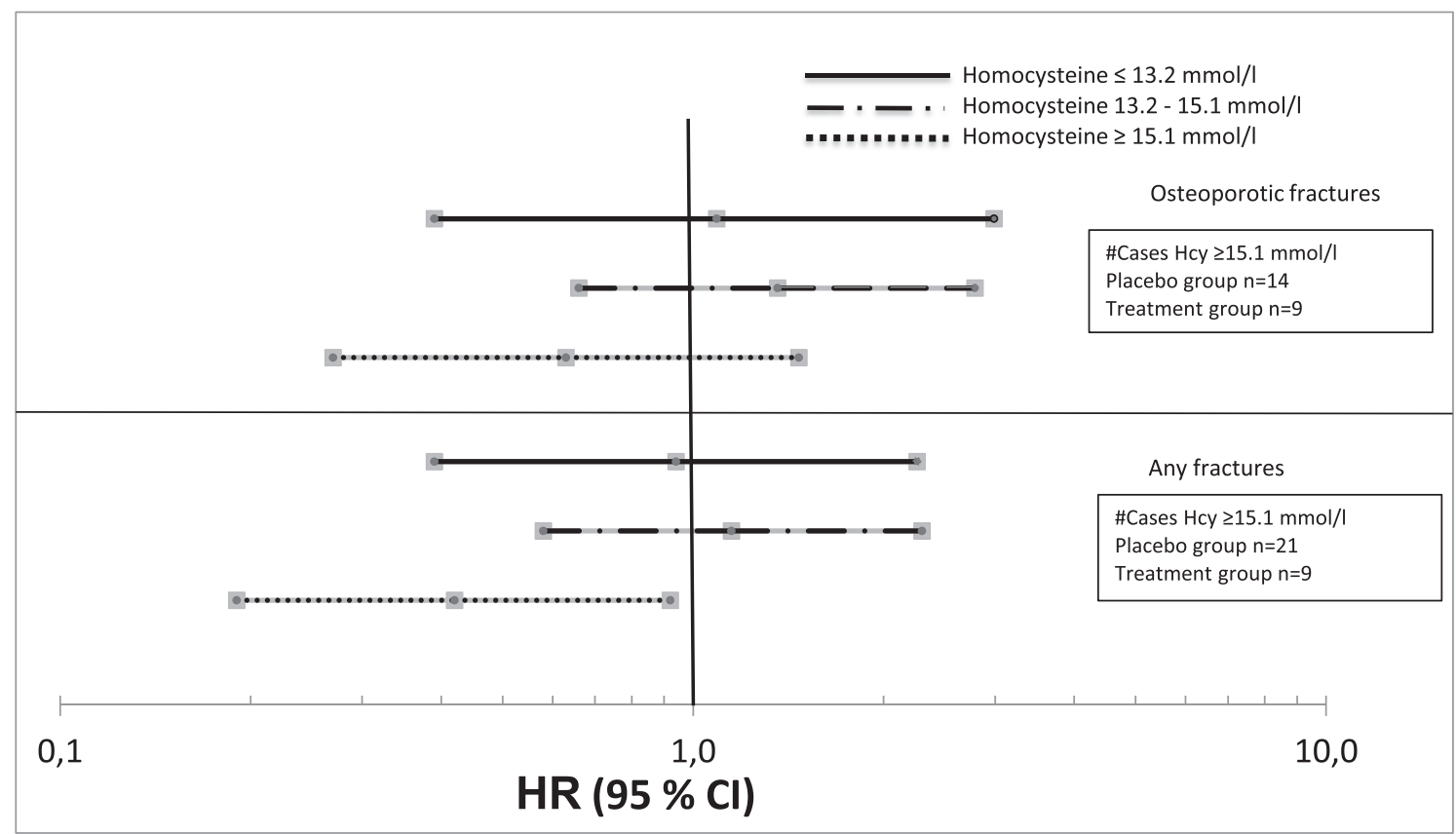

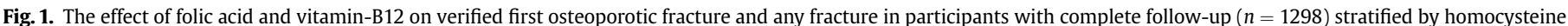
tertiles $(\leq 13.2,13.2-15.1$ and $\geq 15.1 \mathrm{mmol} / \mathrm{l})$ in the adjusted model ( $p$-for interaction $=0.10$ and $p=0.06$ respectively). Hcy $=$ Homocysteine, HR $=$ Hazard Ratio.

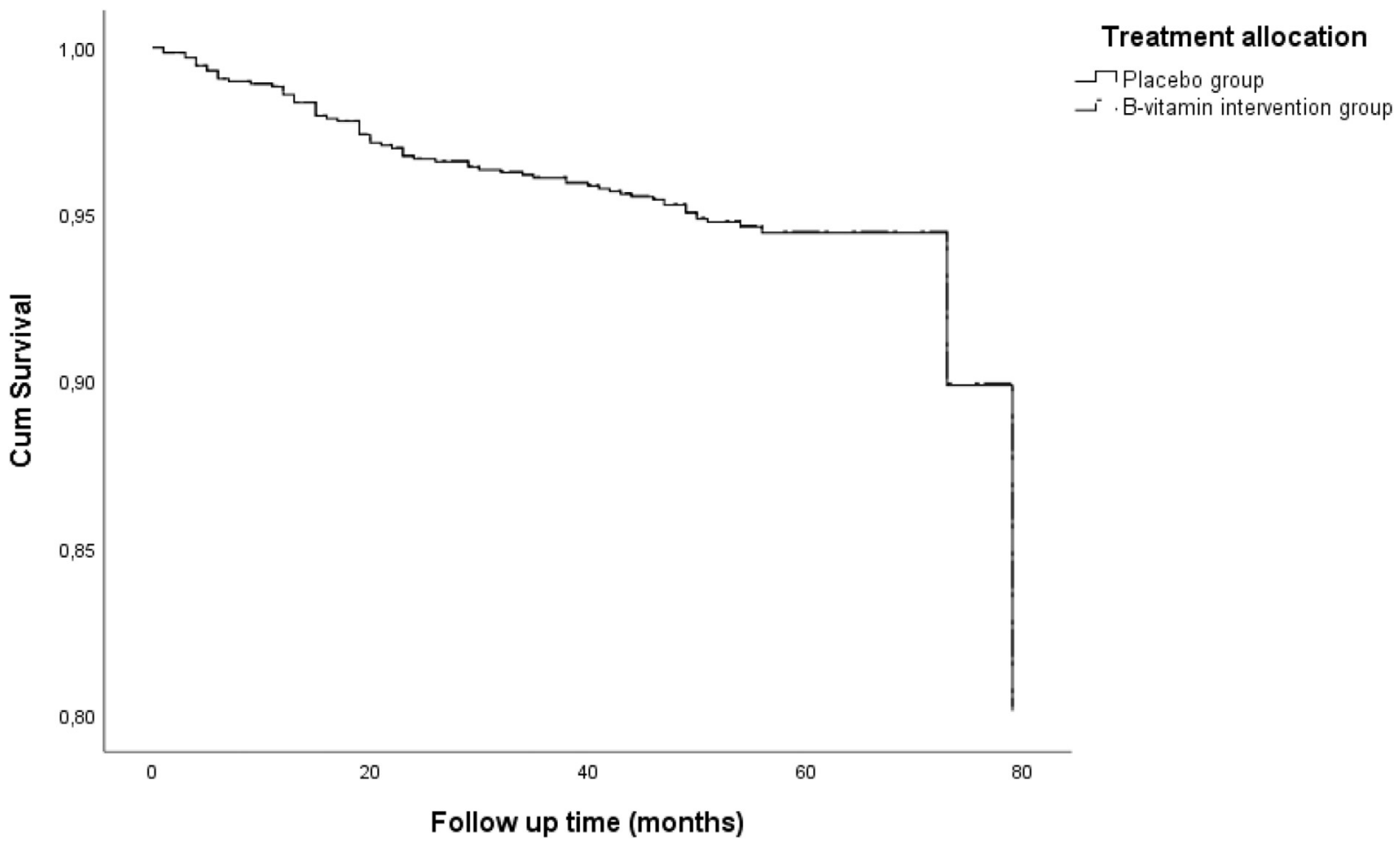

Fig. 2. Kaplan-Meier curve of survival analysis of any type of fractures for the treatment (continue line) and the placebo (dotted line) group.

0.39-1.31 for heart failure and/or cardiac valve disease). After stratification for study center, the intervention showed a higher risk (not significant) of any type of CVD for the participants from Rotterdam than participants from Amsterdam or Wageningen $(\mathrm{OR}=1.36 ; 95 \% \mathrm{CI}: 0.93-2.00, \mathrm{OR}=0.80 ; 95 \% \mathrm{CI}: 0.45-1.42$ and $\mathrm{OR}=0.78 ; 95 \% \mathrm{CI}: 0.44-1.40$ respectively).

\section{Discussion}

The extended follow-up of 5-7 years of a 2-year supplementation with folic acid and vitamin-B12 within a multicenter, randomized, double-blind, placebo-controlled trial in older adults, showed no effect on incidence of first osteoporotic 
Table 3

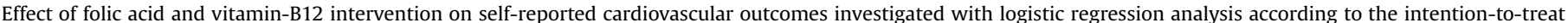
principle in participants with complete follow-up ( $n=1,298)$.

\begin{tabular}{|c|c|c|c|c|c|c|}
\hline \multirow[t]{3}{*}{ Outcome } & \multirow{3}{*}{$\begin{array}{l}\text { No. of cases in placebo } \\
\text { group (cumulative incidence) }\end{array}$} & \multirow{3}{*}{$\begin{array}{l}\text { No. of cases in treatment } \\
\text { group (cumulative incidence) }\end{array}$} & \multicolumn{4}{|c|}{ Effect of the intervention treatment group compared to placebo group } \\
\hline & & & \multicolumn{2}{|l|}{ Model 1} & \multicolumn{2}{|l|}{ Model 2} \\
\hline & & & OR $[95 \% \mathrm{CI}]$ & $p$-Value & OR $[95 \% \mathrm{CI}]$ & $p$-Value \\
\hline Any type CVD & $120(0.88)$ & $130(0.94)$ & $1.06[0.80 ; 1.39]$ & 0.71 & $1.05[0.80 ; 1.39]$ & 0.72 \\
\hline MI & $18(0.33)$ & $20(0.44)$ & $1.07[0.56 ; 2.05]$ & 0.83 & $1.08[0.56 ; 2.05]$ & 0.84 \\
\hline Heart failure and/or heart valve & $42(2.00)$ & $43(2.15)$ & $0.99[0.64 ; 1.54]$ & 0.95 & $1.02[0.66 ; 1.59]$ & 0.98 \\
\hline Angina pectoris & $35(1.94)$ & $41(2.92)$ & $1.14[0.72 ; 1.81]$ & 0.59 & $1.13[0.71 ; 1.80]$ & 0.61 \\
\hline Cerebrovascular disease & $30(0.65)$ & $27(0.69)$ & $0.86[0.51 ; 1.47]$ & 0.59 & $0.85[0.50 ; 1.45]$ & 0.55 \\
\hline
\end{tabular}

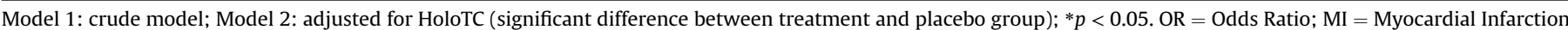
Reference group $=$ placebo group .

or any type of fractures and multiple osteoporotic fractures. However, after stratification, the intervention led to a significantly lower incidence of any fractures in the participants with the highest total baseline homocysteine. Furthermore, the intervention had no effect on the incidence of CVD or cerebrovascular disease.

No overall long-term effect of 2 years of supplementation of folic acid and vitamin-B12 on fracture risk was found in our extended follow-up study. This is in line with the results of recent metaanalysis where no association between homocysteine-lowering treatment with these B-vitamins and the risk of fracture was shown including long term follow-up studies [10]. However, only 2 of the 6 included trials had a longer (extended) follow-up (7 years and 11.1 years) [3,21]. Although the initial B-PROOF trial was also included in this meta-analysis, differences in study design and population with B-PROOF should be noted. The study population of other included trials was younger compared to B-PROOF (62.5-68.8 years). Nevertheless, there was no indication of an age-dependent effect in our current extended follow-up analyses. The baseline total homocysteine concentration of the included participants was also different compared to our study (9.8-13.4 mmol/l versus $14.4 \mathrm{mmol} /$ 1). Interestingly, we found a lower fracture incidence for the group with higher total homocysteine concentration at baseline (>15.1 $\mu \mathrm{mol} / \mathrm{l}$ ). The findings were supported by the tendency toward fracture reduction in the total group, but not by the findings on osteoporotic fractures. Yet the numbers of cases were low in the stratified analysis and for this reason, these explorative findings should be interpreted with caution. Also, the participants in the treatment group with higher baseline homocysteine concentration had a steeper decline of total homocysteine concentration after the supplementation of folic acid and vitamin-B12 than the participants with lower baseline homocysteine concentration suggesting that the effect of the intervention was more pronounced in participants with higher total homocysteine concentration. This is in line with treatment of vitamin D deficiency, where the effect on serum parathyroid hormone concentration is greater when the baseline serum 25hydroxyvitamin D is lower [22]. In general, vitamin supplementation may show a threshold effect, working only in deficient people [23]. In a similar way of reasoning, the effects may soon disappear after discontinuation of supplementation. This follow-up study reports outcomes after a follow-up of 5-7 years, including treatment for 2-3 years only, thus, the effect of supplementation may be disappeared. Besides, from our previous findings of an increased risk of colorectal cancer with B-vitamins supplementation, we do not recommend supplementation of these vitamins in not-deficient general population [18].

It may be speculated that the latter indicates a (intracellular) Bvitamin deficiency [24]. As known, B-vitamins lower total homocysteine concentration and play an important role in the homocysteine metabolism [1]. However, the studies of the relation between high homocysteine concentration and bone show conflicting results [25]. From the previous studies which reported an association between elevated total homocysteine concentration and fracture risk, it remains unclear whether this could be explained by disrupted one-carbon metabolism or whether residual confounding by other physiological and lifestyle factors that associate with hyperhomocysteinemia may play a role [25]. The one-carbon metabolism can be disrupted by vitamin-B12 and folate deficiencies. However, other causes of hyperhomocysteinemia are high intake of methionine, certain diseases (chronic renal failure, hypothyroidism and malignant tumors in the breast, ovary or pancreas) and ingestion of certain drugs [26-29]. However, in our study, vitamin-B12 and folate level was not an effect modifier in the effect of the intervention on fracture risk, suggesting that different levels of vitamin-B12 and folate would not make a difference in the risk of fracture. Our population was also not deficient in B-vitamin measured by different methods (active vitamin-B12, HoloTC and MMA). Since the methods to detect vitamin-B12 and folate deficiency are under debate [30], due to its low biased value of Bvitamin level, the effectiveness of the intervention in the high homocysteine group might be explained by a subclinical deficiency of B-vitamins, that warrants further study.

With regard to the cardiovascular diseases, we found no effect of the intervention with B-vitamins and risk of cardiovascular diseases in this extended follow-up. However, like the initial B-PROOF study we found a lower incidence of cerebrovascular events in the treatment group, but this was not significant. In line with our results, an update of a Cochrane review showed no effect of homocysteine-lowering B-vitamins supplementation compared to placebo on MI, but they did show a small reduced risk of stroke with B-vitamin interventions (vitamin B6, B9 or B12 given alone or in combination compared to placebo $\mathrm{RR}=0.90 ; 95 \% \mathrm{CI}$ : 0.82-0.99) [5]. Also, the recent meta-analysis of Jenkins et al. showed reduced risk of stoke with folic acid alone and B-vitamins with folic acid, B6 and $\mathrm{B} 12$ ( $\mathrm{RR}=0.83 ; 95 \% \mathrm{CI}$ : $0.69-0.93$ for folic acid treatment and $\mathrm{RR}=0.90 ; 95 \% \mathrm{CI}: 0.81-1.00$ for B-complex treatment) [31]. However, these latter results were driven by one large Chinese trial of 20,000 participants.

For the effect of the intervention on CVD and heart failure/cardiac valve disease, the risks were higher in women compared to men, however, the differences were not significant. A possible greater vulnerability of women to folic acid and vitamin-B12 supplementation could be explained by the influences of sex hormones in one-carbon metabolism and the differences between men and women in the expression level of enzymes in this metabolism [32].There is a gap in the knowledge of CVD in different sex and age groups, due to under-representation of women and the older population (because of higher comorbidities) [33]. It has been suggested that there is more variability of the increased risk factors by ageing, related to sex differences that could change between 
middle-aged and elderly adults [33]. In addition, heart failure, occurs mostly in (postmenopausal) women [34]. Thus, probably, the differences in incidence of CVD are due to sex-differences in baseline risk, regardless of the intervention.

\subsection{Strengths and limitations}

A strength of our study is that the B-PROOF trial was initially designed to study fracture risk as primary outcome in an older population. The extended follow-up period allowed us to study long-term effects as well as increase power, and it allowed us to analyse the risk of multiple fractures.

A limitation of our study was that some of the baseline characteristics of the responders were different compared to the total populations and non-responders of the second follow-up questionnaire. The participants who did not return the second questionnaire, were older, and the high mortality and morbidity rate within this age group, may have influenced our results through competing risk bias. Also, the differed in HoloTC and vitamin-B12 levels. So, the results are less powered and need to be interpreted with caution. However, the variables were not different between the treatment and placebo group in responders and nonresponders, respectively, which indicate that the randomisation and internal validity was still intact.

A second limitation is the self-reported cardiovascular events used both in the initial and the extended B-PROOF study. However, the agreement between self-reported and verified events for CVD and cerebrovascular events were more than adequate, respectively 0.89-0.72 (excellent and fair to good agreement). Due to missing of completely verified data, we were not able to do time to event analysis for these outcomes. Additionally, events in the subgroups of CVD (MI, AP, heart failure and cardiac valve disease) as well as cerebrovascular disease group were too small to conduct in depth analysis.

Moreover, there were more participants recruited from Rotterdam than other regions (1,285 participants from Rotterdam and, 857 from Wageningen and 777 from Amsterdam). Other differences in characteristics were found between study centers: participants from Rotterdam had lower 25(OH)D level, lower SES, higher use of vitamin $\mathrm{D}$, folic acid and vitamin-B12 supplements, compared to other regions. Also, the incidence of CVD was already higher at the baseline for the participants from Rotterdam with an extended follow-up. Nonetheless, after stratification by study center, the intervention showed a higher risk of any type of CVD for participants who were recruited from Rotterdam, however, not significant.

Finally, the population with extended follow-up reported a lower use of over the counter vitamin B supplements at FU1 and FU2 compared to the total population at FU1, but the treatment group reported a higher intake of folic acid and vitamin-B12 supplements compared to the placebo group at FU2. Other differences between groups might have arisen after the baseline visit and FU1 (for example drug use and other diseases), which we were unfortunately not able to measure. Furthermore, we have no information about the B-vitamin level and total homocysteine concentration at FU2 to evaluate the effect of the intervention on actual blood biomarkers.

In conclusion, in the extended follow-up of B-PROOF, an overall effect of supplementation of folic acid and vitamin-B12 on fracture risk, CVD and cerebrovascular risk in older individuals with elevated homocysteine concentration was not observed. However, the results of the stratified analyses suggest a reduced fracture risk in individuals with higher total homocysteine concentration. This needs further replication. Currently, we do not recommend supplementation of these B-vitamins in healthy (non-deficient) general population for fracture prevention.

\section{Funding}

The initial B-PROOF study has received funding so far by The Netherlands Organization for Health Research and Development (ZonMw, Grant 6130.0031), the Hague; unrestricted grant from NZO, Zoetermeer; Orthica, Almere; NCHA (Netherlands Consortium Healthy Ageing) Leiden/Rotterdam; Ministry of Economic Affairs, Agriculture and Innovation (project KB-15-004-003), the Hague; Wageningen University, Wageningen; VUmc,Amsterdam; Erasmus Medical Center, Rotterdam.

\section{Conflict of interest}

The authors declare no conflict of interest.

\section{Acknowledgements}

We are grateful to all study participants and co-workers who helped in the organization of the B-PROOF trial. We would particularly like to acknowledge the support of Ms. A. Ham, Dr.; Ms. A.W. Enneman, Dr.; Ms. J.P. van Wijngaarden, Dr.; Mrs. S. Smit, R.N. and Ms. P.H. in 't Veld, MSc., for practical help in the trial.

The sponsors and patients had no role in the design or implementation of the study, data collection, data management, data analysis, data interpretation, or in the preparation, review, or approval of the manuscript. The outcome measures were orally presented in the end of the initial study (2013) by inviting the participants who were interested. Also, the results of the study have been sent to all the study participants.

\section{Appendix A. Supplementary data}

Supplementary data to this article can be found online at https://doi.org/10.1016/j.clnu.2020.07.033.

\section{References}

[1] Selhub J. Homocysteine metabolism. Annu Rev Nutr 1999;19:217-46.

[2] Lowering blood homocysteine with folic acid based supplements: metaanalysis of randomised trials. Homocysteine Lowering Trialists' Collaboration. BMJ 1998;316(7135):894-8.

[3] Stone KL, Lui LY, Christen WG, Troen AM, Bauer DC, Kado D, et al. Effect of combination folic acid, vitamin B6, and vitamin B12 supplementation on fracture risk in women: a randomized, controlled trial. J Bone Miner Res 2017:32(12):2331-8.

[4] Study of the Effectiveness of Additional Reductions in C, Homocysteine Collaborative G, Armitage JM, Bowman L, Clarke RJ, Wallendszus K, et al. Effects of homocysteine-lowering with folic acid plus vitamin B12 vs placebo on mortality and major morbidity in myocardial infarction survivors: a randomized trial. JAMA 2010;303(24):2486-94.

[5] Marti-Carvajal AJ, Sola I, Lathyris D, Dayer M. Homocysteine-lowering interventions for preventing cardiovascular events. Cochrane Database Syst Rev 2017;8:CD006612.

[6] van Wijngaarden JP, Swart KM, Enneman AW, Dhonukshe-Rutten RA, van Dijk SC, Ham AC, et al. Effect of daily vitamin B-12 and folic acid supplementation on fracture incidence in elderly individuals with an elevated plasma homocysteine concentration: B-PROOF, a randomized controlled trial. Am J Clin Nutr 2014;100(6):1578-86.

[7] Kim GS, Kim CH, Park JY, Lee KU, Park CS. Effects of vitamin B12 on cell proliferation and cellular alkaline phosphatase activity in human bone marrow stromal osteoprogenitor cells and UMR106 osteoblastic cells. Metabolism 1996:45(12):1443-6.

[8] Bailey RL, van Wijngaarden JP. The role of B-vitamins in bone health and disease in older adults. Curr Osteoporos Rep 2015;13(4):256-61.

[9] van Wijngaarden JP, Doets EL, Szczecinska A, Souverein OW, Duffy ME, Dullemeijer C, et al. Vitamin B12, folate, homocysteine, and bone health in adults and elderly people: a systematic review with meta-analyses. J Nutr Metab 2013;2013:486186.

[10] Garcia Lopez M, Baron JA, Omsland TK, Sogaard AJ, Meyer HE. Homocysteinelowering treatment and the risk of fracture: secondary analysis of a randomized controlled trial and an updated meta-analysis. JBMR Plus 2018;2(5): 295-303. 
[11] Gommans J, Yi Q, Eikelboom JW, Hankey GJ, Chen C, Rodgers H, et al. The effect of homocysteine-lowering with B-vitamins on osteoporotic fractures in patients with cerebrovascular disease: substudy of VITATOPS, a randomised placebo-controlled trial. BMC Geriatr 2013;13:88.

[12] Sawka AM, Ray JG, Yi Q Josse RG, Lonn E. Randomized clinical trial of homocysteine level lowering therapy and fractures. Arch Intern Med 2007; 167(19):2136-9.

[13] Cole BF, Baron JA, Sandler RS, Haile RW, Ahnen DJ, Bresalier RS, et al. Folic acid for the prevention of colorectal adenomas: a randomized clinical trial. JAMA 2007;297(21):2351-9.

[14] Rose G. Incubation period of coronary heart disease. $\mathrm{Br}$ Med J 1982;284(6329):1600-1.

[15] Llewellyn-Bennett R, Edwards D, Roberts N, Hainsworth AH, Bulbulia R Bowman L. Post-trial follow-up methodology in large randomised controlled trials: a systematic review. Trials 2018;19(1):298.

[16] Lalmohamed A, Welsing PM, Lems WF, Jacobs JW, Kanis JA, Johansson H, et al. Calibration of FRAX (R) 3.1 to the Dutch population with data on the epidemiology of hip fractures. Osteoporos Int 2012;23(3):861-9.

[17] van Wijngaarden JP, Dhonukshe-Rutten RA, van Schoor NM, van der Velde N, Swart KM, Enneman AW, et al. Rationale and design of the B-PROOF study, a randomized controlled trial on the effect of supplemental intake of vitamin B12 and folic acid on fracture incidence. BMC Geriatr 2011;11:80.

[18] Oliai Araghi S, Kiefte-de Jong JC, van Dijk SC, Swart KMA, van Laarhoven HW, van Schoor NM, et al. Folic acid and vitamin B12 supplementation and the risk of cancer: long-term follow-up of the B vitamins for the prevention of osteoporotic fractures (B-PROOF) trial. Cancer Epidemiol Biomarkers Prev 2018.

[19] Amorim LD, Cai J. Modelling recurrent events: a tutorial for analysis in epidemiology. Int J Epidemiol 2015;44(1):324-33.

[20] Therneau TM, Grambsch, Patricia M. Modeling survival data: extending the Cox model. 1st ed.vol. XIV. New York: Springer-Verlag; 2000. p. 350.

[21] Garcia Lopez M, Bonaa KH, Ebbing M, Eriksen EF, Gjesdal CG, Nygard O, et al. $\mathrm{B}$ vitamins and hip fracture: secondary analyses and extended follow-up of two large randomized controlled trials. J Bone Miner Res 2017;32(10):1981-9.

[22] Lips P, Duong T, Oleksik A, Black D, Cummings S, Cox D, et al. A global study of vitamin D status and parathyroid function in postmenopausal women with osteoporosis: baseline data from the multiple outcomes of raloxifene evaluation clinical trial. J Clin Endocrinol Metab 2001;86(3):1212-21.

[23] Sohl E, de Jongh RT, Heymans MW, van Schoor NM, Lips P. Thresholds for serum 25(OH)D concentrations with respect to different outcomes. J Clin Endocrinol Metab 2015;100(6):2480-8.

[24] Joosten E, van den Berg A, Riezler R, Naurath HJ, Lindenbaum J, Stabler SP, et al. Metabolic evidence that deficiencies of vitamin B-12 (cobalamin), folate, and vitamin B-6 occur commonly in elderly people. Am J Clin Nutr 1993;58(4):468-76.

[25] Fratoni V, Brandi ML. B vitamins, homocysteine and bone health. Nutrients 2015;7(4):2176-92.

[26] Kim J, Kim H, Roh H, Kwon Y. Causes of hyperhomocysteinemia and its pathological significance. Arch Pharm Res 2018;41(4):372-83.

[27] Selhub J, Jacques PF, Wilson PW, Rush D, Rosenberg IH. Vitamin status and intake as primary determinants of homocysteinemia in an elderly population. JAMA 1993;270(22):2693-8.

[28] Ham AC, Enneman AW, van Dijk SC, Oliai Araghi S, Swart KM, Sohl E, et al. Associations between medication use and homocysteine levels in an older population, and potential mediation by vitamin B12 and folate: data from the B-PROOF study. Drugs Aging 2014;31(8):611-21.

[29] Alvarez-Sanchez N, Alvarez-Rios AI, Guerrero JM, Garcia-Garcia FJ, RodriguezManas L, Cruz-Chamorro I, et al. Homocysteine levels are associated with bone resorption in pre-frail and frail Spanish women: the Toledo Study for Healthy aging. Exp Gerontol 2018;108:201-8.

[30] Green R. Indicators for assessing folate and vitamin B-12 status and for monitoring the efficacy of intervention strategies. Am J Clin Nutr 2011;94(2): 666S-72S.

[31] Jenkins DJA, Spence JD, Giovannucci EL, Kim YI, Josse R, Vieth R, et al. Supplemental vitamins and minerals for CVD prevention and treatment. J Am Coll Cardiol 2018:71(22):2570-84.

[32] Sadre-Marandi F, Dahdoul T, Reed MC, Nijhout HF. Sex differences in hepatic one-carbon metabolism. BMC Syst Biol 2018;12(1):89.

[33] Trevisan CSG, Veronese N, Maggi S, Manzato E. Gender differences in coronary heart disease in elderly populations. Ital J Gender Spec Med 2017:3(1):3-14.

[34] Bozkurt B, Khalaf S. Heart failure in women. Method Debakey Cardiovasc J 2017;13(4):216-23. 